\title{
Influencia de la hipotiroxinemia materna durante la gestación sobre el desarrollo psicomotor
}

\author{
M. Suárez Rodríguez, C. Azcona San Julián y V. Alzina de Aguilar
}

Departamento de Pediatría. Clínica Universitaria de Navarra. Pamplona. Navarra. España.

\section{Introducción}

La función tiroidea materna durante los primeros meses de embarazo desempeña un papel determinante en el desarrollo del cerebro fetal, porque no existe producción de hormona tiroidea fetal hasta la semana 20.

\section{Material y métodos}

Durante el año 2002 se seleccionó una muestra de 147 mujeres embarazadas en la semana 37 de gestación. Para valorar la función tiroidea de estas gestantes se determinaron las concentraciones séricas de T4 libre y de hormona tiroestimulante (TSH). Posteriormente, se evaluó el desarrollo psicomotor de los hijos de dichas mujeres mediante las escalas McCarthy.

\section{Resultados}

Se obtuvo una mediana de T4 libre de $9,37 \mathrm{pmol} / 1$, y más de la mitad de las gestantes de la muestra presentaron valores por debajo del umbral de hipotiroxinemia. Los hijos de madres con concentraciones de $\mathrm{T} 4 \mathrm{4}$ por debajo del percentil 10 presentaban una puntuación en el índice general cognitivo significativamente más baja que la de los hijos cuyas madres tenían concentraciones séricas de T4 libre normales. Existe una correlación positiva entre el valor de T4 libre materna y el índice general cognitivo $(\mathrm{r}=0,43 ; \mathrm{p}<0,01)$

\section{Conclusiones}

Las concentraciones de T4́ libre materna no sólo son importantes durante los primeros meses de gestación para asegurar un desarrollo adecuado del cerebro fetal, sino durante todo el embarazo.

Palabras clave:

Hipotiroxinemia. Desarrollo psicomotor. T4 libre. Función tiroidea.

\section{INFLUENCE OF MATERNAL HYPOTHYROXINAEMIA DURING PREGNANCY ON PSYCHOMOTOR DEVELOPMENT}

\section{Introduction}

The maternal thyroid function during early pregnancy plays a fundamental role in foetal brain development as synthesis of thyroid hormone does not begin until the $20^{\text {th }}$ week of gestation.

\section{Material and methods}

Throughout the year 2002, 147 women in their $37^{\text {th }}$ week of pregnancy were enrolled for the study. To evaluate their thyroid function, the serum concentrations of free $T 4$ and of TSH were determined. After birth, the psychomotor development of their children was evaluated with the McCarthy scales.

Results

The median value of free $T 4$ was $9.37 \mathrm{pmol} / 1$, being the data obtained from more than half of the sampled women below the hypothyroxinaemia threshold. Children born from mothers with $\mathrm{T} 4$ levels below percentile 10 showed a significantly lower score on the general cognitive index than those whose mothers had normal free $T 4$ serum concentrations. A positive correlation was found between the values of maternal free $T 4$ and the general cognitive index $(r=0.43 ; p<0.01)$.

\section{Conclusions}

The concentrations of maternal free $\mathrm{T} 4 \mathrm{are}$ important, not only during the first months of pregnancy, but all along the process to ensure adequate development of the foetal brain.

Key words:

Hypothyroxinaemia. Psychomotor development. Free T4. Thyroid function.

Correspondencia: Dra. M. Suárez Rodríguez.

Departamento de Pediatría

Clínica Universitaria de Navarra.

Avda. Pío XII, s/n. 31008 Pamplona. España.

Correo electrónico: msr1070@hotmail.com 


\section{INTRODUCCIÓN}

A lo largo de la historia, la deficiencia de yodo ha causado graves alteraciones mentales a millones de personas, debido a sus efectos sobre el cerebro en desarrollo. Todavía hoy, el aporte insuficiente de este oligoelemento es la primera causa de retraso mental evitable en el mundo ${ }^{1,2}$

Hasta la última mitad de la década de 1960 existían diversas hipótesis contradictorias sobre la existencia o no de transferencia de hormonas tiroideas de la madre al feto y sobre su posible relevancia para el desarrollo del cerebro del niño. En la actualidad se ha aceptado que existe un paso transplacentario de hormonas tiroideas maternas al feto desde las primeras semanas de gestación ${ }^{3,4}$, en especial de T4 y, en mucha menor medida, de T3. Así, casi toda la T3 presente en los receptores nucleares de la corteza cerebral fetal desde el primer trimestre de embarazo se genera por la desyodación local de la T4 de origen materno 5 . De esta manera, las concentraciones séricas bajas de T4 libre materna desempeñan un papel fundamental en el desarrollo neurológico del feto, sobre todo durante el primer trimestre de gestación, porque no existe producción de hormona tiroidea fetal hasta la semana 20 de embarazo ${ }^{2,6-10}$.

En 1990, la Declaración Mundial para la Supervivencia, Protección y Desarrollo de la Infancia identifica la necesidad de eliminar las disfunciones por deficiencia de yodo y la incluye entre los objetivos a cumplir antes del año $2000^{11}$. Posteriormente, todos los organismos internacionales implicados en la protección y defensa de la infancia (OMS, UNICEF, FAO, ICCIDD) han ratificado el derecho de cada niño a un adecuado aporte de yodo para asegurar su normal desarrollo. De este hecho se desprende el derecho que cada madre tiene a recibir una nutrición rica en dicho oligonutriente que asegure el desarrollo mental normal del hijo no nacido ${ }^{5,11}$.

En estudios recientes sobre la prevalencia mundial del déficit de yodo se ha observado que la carencia de este oligoelemento representa un problema de salud pública en 54 países, mientras que sólo 43 han alcanzado una situación óptima en cuanto a su aporte ${ }^{12}$. Se estimó que, en el mundo, un 36,5\% (aproximadamente 285 millones de niños) de los escolares no consumían la cantidad necesaria del micronutriente, porcentaje que oscila entre el 10,1\% en América y el 59,9\% en Europa ${ }^{12}$.

La ingestión diaria de yodo recomendada es de $30 \mu \mathrm{g}$ en recién nacidos prematuros, $90 \mu \mathrm{g}$ desde el nacimiento hasta los 6 años, $120 \mu \mathrm{g}$ hasta la pubertad, $150 \mu \mathrm{g}$ para los adultos ${ }^{13}$ y 250-500 $\mu \mathrm{g}$ durante el embarazo y la lactancia ${ }^{5,14,15}$. Por tanto, durante los períodos de lactancia y gestación los requerimientos de yodo son máximos, ya que se duplican las necesidades con respecto a las que presenta un individuo adulto. Las mujeres que se encuentran en dicha situación son, por esta razón, consideradas como poblaciones de alto riesgo de presentar un déficit de dicho elemento.
El aumento de los requerimientos de yodo durante el embarazo se debe a los cambios hormonales que acontecen durante la gestación. El organismo materno en situación de déficit de yodo va a sufrir una estimulación excesiva de su glándula tiroides, que corre el peligro de no ajustarse adecuadamente a dichas demandas. El patrón analítico en esta situación presentará las siguientes características: concentraciones séricas de T4 libre por debajo del percentil 10, lo que se conoce como hipotiroxinemia, concentraciones séricas de hormona tiroestimulante (TSH) dentro de los límites de la normalidad, relación T3/T4 aumentada debido a una secreción preferencial de T3 y concentraciones de TBG sérica aumentadas $^{11,16}$

Estudios experimentales han demostrado que la hipotiroxinemia materna en etapas precoces del desarrollo (previas a la semana 20 de gestación) determina en el feto alteraciones irreversibles en la citoarquitectura de la corteza somatosensorial y del hipocampo, porque se altera el proceso de migración neuronal ${ }^{6,17}$. Estas lesiones no tienen traducción clínica perinatal, sino que se manifiestan más tarde, durante los primeros años de vida y en la edad escolar. En los estudios de seguimiento de los hijos de madres con hipotiroxinemia en el primer trimestre de gestación, se ha encontrado un descenso en las puntuaciones de la escala de Bayley (escala de medida de desarrollo psicomotor), en especial en los tests que valoran la coordinación visuomotora, la manipulación, la comprensión en la relación de objetos, la imitación y el desarrollo del lenguaje temprano ${ }^{6}$.

Más recientemente, en estudios realizados en dos ciudades italianas, se ha descrito la asociación existente entre la hipotiroxinemia materna durante el embarazo y el trastorno por déficit de atención e hiperactividad en la descendencia ${ }^{18}$.

El objetivo de este estudio ha sido determinar la relación entre los valores de T4 libre maternos durante el tercer trimestre de gestación y el desarrollo psicomotor de la descendencia en un área sin déficit de yodo.

\section{MATERIAL y MÉTODOS}

\section{Selección de los sujetos}

Se incluyeron en el estudio 147 mujeres que se encontraban en el tercer trimestre de gestación (semana 37), controladas en el departamento de ginecología de nuestro centro durante el año 2002.

Como criterios de exclusión se utilizaron los siguientes: diagnóstico de enfermedad tiroidea conocida antes del embarazo, mujeres afectadas de alguna enfermedad digestiva que conllevara una malabsorción intestinal y mujeres en tratamiento con fármacos que alteraran el metabolismo de las hormonas tiroideas.

Posteriormente se incluyeron los hijos de dichas mujeres. 
Se obtuvo un consentimiento informado firmado de todas las gestantes que participaron en el estudio.

\section{Función tiroidea materna}

En la semana 37 de gestación, coincidiendo con la última revisión previa al parto, se obtuvo una muestra de sangre de las embarazadas. Dichas muestras fueron procesadas por el laboratorio de bioquímica del hospital y se midieron las concentraciones séricas de TSH y T4 libre mediante inmunoanálisis enzimático de micropartículas (MEIA). A algunas de las gestantes que presentaban hipotiroxinemia se les realizó, además, una determinación sérica de tiroglobulina, T3 libre y anticuerpos antitiroglobulina mediante radioinmunoanálisis (RIA) y de anticuerpos antiperoxidasa mediante enzimoinmunoanálisis (ELISA).

Como valores de referencia para las diferentes hormonas se utilizaron los del laboratorio mencionado anteriormente.

\section{Evaluación del desarrollo psicomotor}

Los hijos de las mujeres de la muestra fueron evaluados entre los 42 y los 60 meses de vida mediante las escalas de McCarthy de aptitudes y psicomotricidad para niños $(\mathrm{MSCA})^{19}$. Este método de evaluación permite obtener puntuaciones o índices, derivados de una observación sistemática, de diferentes conductas cognitivas y motoras. El MSCA contiene 18 tests independientes que evalúan las aptitudes del sujeto en ciertas áreas importantes. Los tests han sido agrupados en seis escalas: verbal, perceptivomanipulativa, numérica, general cognitiva o índice general intelectual, memoria y motricidad.

El contenido de las tres primeras escalas no se solapa y cuando se consideran conjuntamente constituyen la escala general cognitiva o índice general intelectual. Esta escala está constituida, por tanto, por todos los tests que forman las escalas verbal, perceptivo-manipulativa y numérica. Todas estas tareas son de naturaleza cognitiva y, en su conjunto, permiten una evaluación de los procesos mentales generales del sujeto. Sólo existen tres tests de las escalas MSCA (coordinación de piernas, coordinación de brazos y acción imitativa) no incluidos en la escala general cognitiva, porque implican más una actividad motora que una aptitud cognitiva.

Con el índice general cognitivo obtenido por un niño se evalúa su nivel intelectual en relación con el de otros sujetos de su misma edad cronológica. Su media es 100 y su desviación típica 16, y los parámetros utilizados para definir los cocientes intelectuales coinciden con los obtenidos en otros tests psicológicos, aunque en las escalas MSCA no se usa el concepto de cociente intelectual.

\section{Análisis estadístico}

Para el análisis estadístico se utilizó el paquete de SPSS versión 15.0 para Windows. Por un lado, se realizó un análisis descriptivo de las variables utilizando la media y la desviación típica para aquellas variables que seguían una distribución normal, y la mediana y el rango intercuartílico para las que no presentaban dicho patrón. Por otro lado, se llevó a cabo una correlación de Pearson para determinar la posible asociación entre el desarrollo psicomotor de los niños y la función tiroidea materna.

\section{Resultados}

\section{Descripción de la muestra}

Se trata de una muestra de 147 gestantes en la semana 37 de embarazo, de las cuales el 45,5\% (67 mujeres) eran primigestas, frente al 55,5\% restante ( 80 mujeres) que ya habían tenido descendencia. Estas últimas habían tenido un embarazo previo normal. La edad de las mujeres estaba comprendida entre los 22 y los 43 años, con una media de 32. Dichas gestantes no presentaban antecedentes médicos reseñables y ninguna de ellas tenía historia de enfermedad tiroidea previa.

\section{Función tiroidea}

Como ya se ha mencionado, para valorar la función tiroidea materna se determinaron inicialmente la TSH y la T4 libre. Como valores de referencia se tomaron los de nuestro laboratorio, de manera que consideramos como normal una TSH comprendida entre 0,2 y $5 \mu \mathrm{U} / \mathrm{ml}$ y una T4 libre comprendida entre 9,5 y 23,9 pmol/1.

En función de estos valores, 7 de las 147 mujeres que iniciaron el estudio (4,8\%) fueron excluidas por presentar hipotiroidismo no diagnosticado antes del embarazo (TSH superior a $5 \mu \mathrm{U} / \mathrm{ml}$ y T4 libre inferior a 9,5 pmol/1). De las 140 participantes restantes, la T4 libre se determinó en 136 (en las otras cuatro hubo un error al hacer la petición y se obtuvieron los valores de T4 total) y la TSH en 139 (una fue excluida por mala extracción de la muestra). Se obtuvo una mediana de T4 libre de 9,37 pmol/1 $(8,29 ; 10,47)$, y 74 de las gestantes $(54,41 \%)$ presentaron valores por debajo del umbral de hipotiroxinemia. La mediana de los valores de TSH fue de 1,43 $\mu \mathrm{U} / \mathrm{ml}(1,07$; $1,88)$ y todas la participantes tuvieron concentraciones inferiores a $5 \mu \mathrm{U} / \mathrm{ml}$ (tabla 1 ).

En las 74 mujeres con hipotiroxinemia se determinaron la T3 libre y los anticuerpos antitiroideos de 26, obteniéndose en un 95\% de ellas valores de T3 libre dentro de los límites normales para nuestro laboratorio (2,2-5,3 pmol/1), con un valor medio de esta hormona de $2,84 \mathrm{pmol} / \mathrm{l}(0,67)$. Los anticuerpos antitiroideos, tanto antiperoxidasa como antitiroglobulina, resultaron negativos en todas ellas.

\section{Desarrollo psicomotor}

Se evaluaron 56 niños con edades comprendidas entre los 42 y los 60 meses. De ellos, 28 eran varones (50\%) y 28 , mujeres (50\%). Se dividió la muestra en dos grupos 


\begin{tabular}{l}
\hline TABLA 1. Estadísticos descriptivos de la muestra de gestantes \\
\begin{tabular}{|lcccccccc|}
\hline \multicolumn{1}{|c}{ Variable } & Media & Desviación típica & Mínimo & Máximo & Mediana & $\mathbf{P}_{\mathbf{2 5}}$ & $\mathbf{P}_{\mathbf{7 5}}$ \\
\hline Edad (años) & 32,8 & 3,98 & 22 & 43 & 33 & 30 & 36 & \\
T4 libre (pmol/l) & 9,46 & 1,62 & 5,06 & 16,59 & 9,37 & 8,29 & 10,47 \\
TSH ( $\boldsymbol{\mu} \mathrm{U} / \mathrm{ml})$ & 1,51 & 0,68 & 0,04 & 4,31 & 1,43 & 1,07 & 1,88 \\
T3 libre (pmol/l) & 2,84 & 0,67 & 1,23 & 4,26 & 2,96 & 2,50 & 3,21 \\
\hline
\end{tabular}
\end{tabular}

TSH: hormona tiroestimulante.

según el valor de T4 libre materna se encontrara por encima o por debajo del percentil 10 (grupos 1 y 2, respectivamente), de tal manera que el grupo 1 estaba compuesto por 23 niños y el grupo 2 por 29 niños. De los 4 niños restantes no teníamos el valor de T4 libre de las madres.

La media del índice general cognitivo para el grupo 1 (T4 > p10) fue de $115,17(5,77)$ mientras que para el grupo 2 (T4< p10) dicho valor fue de 108,72 (5,63), diferencia entre los dos grupos que fue altamente significativa $(\mathrm{p}<0,01)$ (fig. 1).

Existe una correlación positiva entre el valor de T4 libre materna y el índice general cognitivo $(r=0,43 ; \mathrm{p}<0,01)$ (fig. 2).

\section{Discusión}

Los principales cambios que tienen lugar en la función tiroidea durante la gestación se deben al aumento de los requerimientos de las hormonas sintetizadas por esta glándula endocrina, que ya comienzan en el primer trimestre de embarazo. Este incremento de las necesidades sólo se puede suplir con una mayor producción de hormonas, que depende directamente de la cantidad de yodo ingerido en la dieta. Cuando existe un déficit en el aporte de este oligoelemento, el organismo tiene dificultades para mantener los cambios fisiológicos secundarios a la gestación, y éstos son reemplazados progresivamente por alteraciones patológicas, tanto más graves cuanto mayor sea la deficiencia de yodo ${ }^{18-20}$.

El patrón analítico de las participantes se caracteriza porque la mayoría de ellas presentan concentraciones séricas de T4 libre por debajo de los límites considerados como normales, manteniéndose, sin embargo, las concentraciones de TSH dentro de la normalidad. Estos datos son característicos de las mujeres gestantes con un consumo deficitario de yodo.

Se han realizado diversos estudios de seguimiento de hijos de madres con hipotiroxinemia, de entre los que destaca el de Pop et $\mathrm{al}^{6}$, quienes investigaron la relación existente entre las concentraciones séricas de T4 libre materna durante el embarazo y el desarrollo psicomotor del niño en un área con una aporte suficiente de yodo. Para ello estudiaron a 291 gestantes en las que se determinaron la TSH, la T4 libre y los anticuerpos anti-TPO a las 12 y 32 semanas de gestación, 4 semanas después del

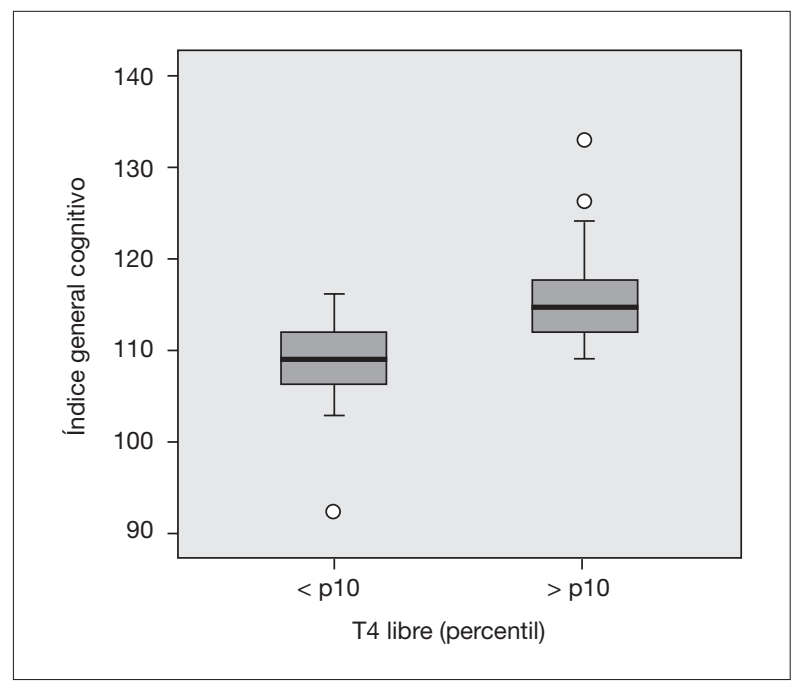

Figura 1. Comparación de la puntuación media del indice general cognitivo en función de los valores séricos de T4 libre materna.

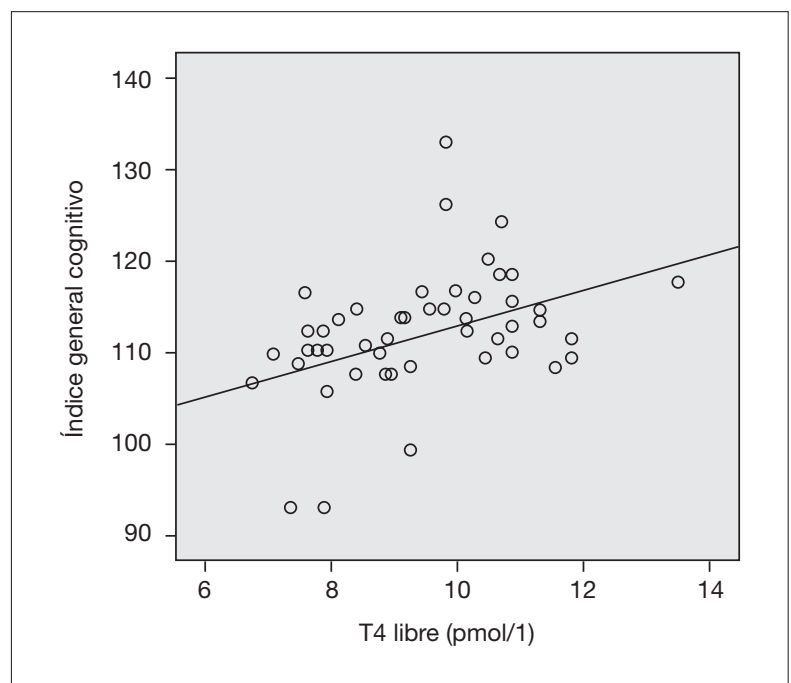

Figura 2. Correlación positiva entre los valores de T4 libre materna y el indice general cognitivo $(r=0,43$; $p<0,01$ ).

parto y, posteriormente, a intervalos de 8 semanas hasta las 36 semanas posteriores al parto. El desarrollo psicomotor de los hijos de estas mujeres se evaluó a los 10 me- 
ses de edad mediante la escala de Bayley de desarrollo infantil. Este estudio llega a la conclusión de que las concentraciones de T4 libre materna por debajo del percentil 10 en la semana 12 de gestación se asocian con un descenso en las puntuaciones de los tests de desarrollo psicomotor. Sin embargo, no pudo demostrarse que los valores de T4 libre a las 32 semanas de gestación estuviesen relacionados con un menor desarrollo psicomotor de los niños a los 10 meses de vida ${ }^{6}$.

Otro estudio similar fue llevado a cabo entre enero de 1997 y abril de 1998 por Kooistra et $\mathrm{al}^{17}$, con la diferencia de que estos investigadores evaluaban el desarrollo de los niños a las 3 semanas de vida mediante el Neonatal Behavioral Assessment Scale (NBAS). Encontraron que hijos de madres con hipotiroxinemia en la semana 12 de gestación obtenían puntuaciones menores en NBAS que los niños nacidos de madres con concentraciones séricas normales de T4 libre, y llegaron a la conclusión de que las concentraciones séricas bajas de T4 libre durante las primeras semanas de gestación conllevan una alteración en el desarrollo cerebral del feto. Asimismo, mediante una regresión lineal demostraron que las concentraciones de T4 libre maternas en el primer trimestre se relacionan con un mejor desarrollo psicomotor del niño, aunque no encontraron dicha correlación con los valores de T4 libre y TSH en el segundo y en el tercer trimestres de gestación ${ }^{17}$.

En el presente estudio no disponemos de las concentraciones séricas de T4 libre materna durante el primer trimestre de gestación, sin embargo sí encontramos una diferencia significativa entre los índices generales cognitivos de los hijos de madres con hipotiroxinemia en la semana 37 y los de mujeres con función tiroidea normal en dicha semana. Quizás esto se deba a que estas gestantes ya presentaban valores de T4 libre por debajo de los requeridos para el correcto desarrollo cerebral fetal en el primer trimestre de embarazo.

En este estudio se demuestra, además, que existe una asociación entre las concentraciones de T4 libre y el desarrollo psicomotor, de manera que a mayor concentración sérica de T4 libre materna, mayor puntuación del índice general cognitivo en la escala MSCA.

En el año 2004, en un estudio realizado en el norte de Sicilia se describió que el 68,8\% de la descendencia de las madres que habían presentado hipotiroxinemia en los primeros meses de embarazo presentaban un trastorno por déficit de atención e hiperactividad, además de obtener coeficientes intelectuales significativamente menores que los hijos de madres con función tiroidea nor$\mathrm{mal}^{18}$.

En conclusión, en este estudio se demuestra que no sólo son importantes los valores de T4 libre materna durante los primeros meses de gestación para asegurar un desarrollo adecuado del cerebro fetal, sino durante todo el embarazo, por lo que nos deberíamos plantear los siguientes retos en la práctica clínica:

1. Asegurar una ingestión de yodo durante el embarazo y la lactancia de unos 250-300 $\mu \mathrm{g}$ al día. En nuestra población, esto sólo se puede conseguir suplementando profilácticamente a toda gestante con yoduro potásico exógeno ${ }^{14,21}$. Dicho suplemento debería ser administrado incluso algunos meses antes de la concepción. En este sentido, hay que tener presente que cuando el déficit se ha establecido desde mucho tiempo antes, se necesitan aproximadamente 3 meses para conseguir una normalización de la función tiroidea tras la administración de suplementos de yodo ${ }^{11,14}$.

2. Por otra parte, sería deseable incluir la determinación sistemática de T4 libre y anticuerpos antitiroideos durante el embarazo. De esta manera podría conocerse la prevalencia de la hipotiroxinemia y del hipotiroidismo clínico y subclínico existente en la población gestante. El momento ideal para estas determinaciones todavía está por establecerse pero, al menos, debería hacerse al inicio del embarazo, coincidiendo con la primera visita al ginecólogo y al final del tercer trimestre de gestación.

\section{Bibliografía}

1. Arena J, Emparanza JI. Acerca de los indicadores de la deficiencia nutricional de yodo. An Pediatr (Barc). 2003;59: 299-301.

2. Pérez-López FR. Iodine and thyroid hormones during pregnancy and postpartum. Gynecol Endocrinol. 2007;23:414-28.

3. Morreale de Escobar G, Obregón MJ, Escobar del Rey F. Maternal thyroid hormones early in pregnancy and fetal brain development. Best Pract Res Clin Endocrinol Metab. 2004;18: $225-48$.

4. Morreale de Escobar G, Obregón MJ, Escobar del Rey F. Role of thyroid hormone during early brain development. Eur J Endocrinol. 2004;151:25-37.

5. Berbel P, Obregón MJ, Bernal J, Escobar del Rey F, Morreale de Escobar G. Iodine supplementation during pregnancy: a public health challenge. Trends Endocrinol Metab. 2007;18: $338-43$.

6. Pop VJ, Kuijpens JL, Van Baar AL, Verkerk G, Van Sons MM, De Vijlder JJ, et al. Low maternal free thyroxine concentrations during early pregnancy are associated with impaired psychomotor development in infancy. Clinical Endocrinol (Oxf). 1999;50:149-55

7. Morreale de Escobar G, Obregón MJ, Escobar del Rey F. Is neuropsychological development related to maternal hypothyroidism or to maternal hypothyroxinemia? J Clin Endocrinol Metab. 2000;85:3975-87.

8. Haddow JE, Palomaki GE, Allan W, Williams J, Knight G, Gagnon J, et al. Maternal Thyroid deficiency during pregnancy and subsequent neuropsychological development of the child. N Engl J Med. 1999;341:549-55.

9. Smallridge R, Ladenson P. Hypothyroidism in pregnancy: consequences to neonatal health. J Clin Endocrinol Metab. 2001; 86:2349-53. 
10. Glinoer D, Delange F. The potential repercussions of maternal, fetal and neonatal hypothyroxinemia on the progeny. Thyroid. 2000;10:871-87.

11. Velasco López I. Anomalías prenatales asociadas a la deficiencia de yodo. Prog Diag Trat Prenat. 2005;17:123-8.

12. Andersson M, Takkouche B, Egli I, Allen HE, Benoist B. Current global iodine status and progress over the last decade towards the elimination of iodine deficiency. Bull World Health Organ. 2005;83:518-25.

13. Delange F, Bürgi H, Chen ZP, Dunn JT. World status of monitoring iodine deficiency disorders control programs. Thyroid. 2002;12:915-24.

14. Soler Ramón J, Aguilar Diosdado M, Gallo M, Martull P, Aguayo J, Arenas MJ, et al. Manifiesto sobre la erradicación de la deficiencia de yodo en España. Prog Diag Trat Prenat. 2005;17:109-10.

15. Abalovich M, Amino N, Barbour LA, Cobin R, De Groot L, Glinoer D, et al. Clinical Practice Guideline. Management of thyroid dysfunction during pregnancy and postpartum: an endocrine society clinical practice guideline. J Clin Endocrinol Metab. 2007;92:1-47.
16. Glinoer D. The regulation of thyroid function during normal pregnancy: Importance of the iodine nutrition status. Best Pract Res Clin Endocrinol Metab. 2004;18:133-52.

17. Kooistra L, Crawford S, Van Baar AL, Brouwers EP, Pop VJ. Neonatal effects of maternal hypothyroxinemia during early pregnancy. Pediatrics. 2006;117:161-7.

18. Vermiglio F, Lo Presti V, Moleti M, Sidoti M, Tortorella G, Scaffidi $G$, et al. Attention deficit hyperactivity disorders in the offspring of mothers exposed to mild-moderate iodine deficiency: A possible novel iodine deficiency disorder in developed countries. J Clin Endocrinol Metab. 2004;89: 6054-60.

19. McCarthy D. Características generales. MSCA, Escalas McCarthy de aptitudes y psicomotricidad para niños. Manual. 7. ed. Madrid: TEA Ediciones; 2004. p. 9-22.

20. Glinoer D. The regulation of thyroid function in pregnancy: pathways of endocrine adaptation from physiology to pathology. Endocr Rev. 1997;18:404-33.

21. Glinoer D. What happens to the normal thyroid during pregnancy? Thyroid. 1999;7:631-5. 